\title{
COLLECTIONS OF SUBSETS WITH THE SPERNER PROPERTY
}

\author{
BY
}

JERROLD R. GRIGGS

\begin{abstract}
Let $X=\{1, \ldots, n\}$ and $Y=\{1, \ldots, k\}, k \leqslant n$. Let $C(n, k)$ be the subsets of $X$ which intersect $Y$, ordered by inclusion. Lih showed that $C(n, k)$ has the Sperner property. Here it is shown that $C(n, k)$ has several stronger properties. A nested chain decomposition is constructed for $C(n, k)$ by bracketing. $C(n, k)$ is shown to have the LYM property. A more general class of collections of subsets is studied: Let $X$ be partitioned into parts $X_{1}, \ldots, X_{m}$, let $I_{1}, \ldots, I_{m}$ be subsets of $\{0,1, \ldots, n\}$, and let $P=\left\{Z \subset X|| Z \cap X_{i} \mid \in I_{i}, 1<i<m\right\}$. Sufficient conditions on the $I_{i}$ are given for $P$ to be LYM, or at least Sperner, and examples are provided in which $\boldsymbol{P}$ is not Sperner. Other results related to Sperner's theorem, the Kruskal-Katona theorem, and the LYM inequality are presented.
\end{abstract}

In 1928 Sperner [18] showed that if $\mathcal{F}$ is a collection of subsets of $X=$ $\{1, \ldots, n\}$ such that no set in $\mathscr{F}$ contains any other, then

$$
|\mathcal{F}| \leqslant\left(\begin{array}{c}
n \\
{\left[\frac{1}{2} n\right\rceil}
\end{array}\right)
$$

This bound is best-possible and is attained if and only if $\mathscr{F}$ consists of all subsets of $X$ of size $\left\lfloor\frac{1}{2} n\right\rfloor$ or all subsets of size $\left\lceil\frac{1}{2} n\right\rceil .(\lfloor a\rfloor$ and $\lceil a\rceil$ are, respectively, the greatest integer $\leqslant a$ and the least integer $\geqslant a$.) Lih [15] recently discovered this generalization of Sperner's theorem: With $X$ as above, let $Y$ be a $k$-element subset of $X$, say $Y=\{1, \ldots, k\}$. Again let $\mathscr{F}$ be a collection of subsets of $X$ with no set in $\mathscr{F}$ containing any other, but with the additional property that each set in $\mathscr{F}$ has a nonempty intersection with $Y$. Then

$$
|\mathscr{F}| \leqslant\left(\begin{array}{c}
n \\
\left\lceil\frac{1}{2} n\right\rceil
\end{array}\right)-\left(\begin{array}{c}
n-k \\
\left\lceil\frac{1}{2} n\right\rceil
\end{array}\right) .
$$

Equality is attained here for example if $\mathcal{F}$ consists of all subsets of $X$ of size $\left\lceil\frac{1}{2} n\right\rceil$ which intersect $Y$.

Let $C(n, k)$ denote the collection of all subsets of $X$ which intersect $Y$, ordered by inclusion. Lih's result is that $C(n, k)$ has the Sperner property. Following a review of the terminology of extremal set theory in $\S 1$, we show in $\S 2$ that $C(n, k)$ has a stronger property: It is a nested chain order.

In $\$ 3$ we show that $C(n, k)$ has the LYM property, which is also stronger than the Sperner property. We broaden this by looking at collections of all subsets of $X$ with specified intersection sizes with each part of a partition of $X$. Specifically,

Received by the editors August 25, 1980 and, in revised form, January 20, 1981.

1980 Mathematics Subject Classification. Primary 05A05; Secondary 06A10.

(C) 1982 American Mathematical Society 0002-9947/82/0000-0775/\$05.25 
suppose that $X$ is partitioned into parts $X_{1}, \ldots, X_{m}$, and let $I_{1}, \ldots, I_{m}$ be subsets of $\{0,1, \ldots, n\}$. Let

$$
P=\left\{Z \subset X|| Z \cap X_{i} \mid \in I_{i}, 1 \leqslant i \leqslant m\right\},
$$

ordered by inclusion. For instance, if $m=2, X_{1}=Y, X_{2}=X-Y, I_{1}=$ $\{1, \ldots, n\}$, and $I_{2}=\{0,1, \ldots, n\}$, we get $P=C(n, k)$. We employ the product theorem for LYM posets to show that $P$ is LYM and has logarithmically concave Whitney numbers whenever the sets $I_{i}$ are all arithmetic progressions. Wareham [19] has discovered a new proof of this result from a different perspective which employs networks and flow-morphisms.

Additional sufficient conditions on the $I_{i}$ for $P$ to be Sperner are derived in $\$ 4$ with the assistance of other tools: The product theorems for symmetric chain orders and for posets with property $S$.

Counterexamples to several further generalizations of the results here are given in $\S 5$. For example, it is shown that without any restrictions on the $I_{i}, P$ above need not have the LYM property nor even the Sperner property. We also present a collection of subsets of $X$ of fixed size such that the filter generated by them is not Sperner. This is a counterexample to a conjecture of Lih.

A different proof that $C(n, k)$ is LYM is presented in $\$ 6$ which employs the Kurskal-Katona theorem. This approach does not yield the broad results of $\S 3$, but the proof is of interest in itself: A new lower bound is given for the size of the collection $S^{*}$ of $(m+1)$-subsets of $X$ which each contain at least one of a collection $S$ of $m$-subsets of $X$.

Sperner found all maximum-sized antichains $\mathscr{F}$ of subsets of $X$ : He found that all sets in such an $\mathscr{F}$ have the same size. This result is extended to $C(n, k)$ in $\S 7$ by a proof which generalizes Sperner's proof.

$\S 8$ concludes the paper with a new LYM-style inequality for antichains in $C(n, k)$ that for $k=n$ is the usual LYM inequality for antichains of subsets of $X$.

The following theorems contain independent proofs that $C(n, k)$ is Sperner: Theorems 2.1, 3.2, 4.4, 6.1, and 7.1.

1. Terminology. A finite poset (partially ordered set) $P$ is ranked (graded) if there exists a function $r: P \rightarrow\{0,1,2, \ldots\}$ such that $r(x)=0$ for minimal elements $x$ in $P$ and $r(y)=r(z)+1$ if $y$ covers $z$ in $P . r(x)$ is the rank of $x$, and the rank of $P$ is the maximum rank of the elements of $P . C(n, k)$ is ranked and for $A \in C(n, k)$, $r(A)=|A|-1$. If $P$ is ranked, the number of elements of rank $j$ is called the $j$ th Whitney number, denoted by $N_{j}(P)$.

$B_{n}$ denotes the Boolean algebra of order $n$, which consists of all $2^{n}$ subsets of $X=\{1, \ldots, n\}$, ordered by inclusion.

A chain (respectively, antichain) is a totally ordered (unordered) subset of $P$. $d_{1}(P)$ denotes the width of $P$ which is the size of the largest antichain in $P$. A ranked poset $P$ has the Sperner property if $d_{1}(P)=\max _{j} N_{j}(P)$. For any $k$ a $k$-family is the union of $k$ antichains in $P . d_{k}(P)$ is the size of the largest $k$-family in $P$. A ranked poset $P$ has property $S$ [7] (or the strong Sperner property [17]) if for all $k d_{k}(P)$ is the sum of the $k$ largest Whitney numbers in $P$. 
A ranked poset $P$ has the $L Y M$ property if every antichain $A \subseteq P$ satisfies this inequality:

$$
\sum_{x \in A} \frac{1}{N_{r(x)}(P)} \leqslant 1 .
$$

If $\boldsymbol{P}$ has the LYM property, then it has property $S$ and hence the Sperner property.

A regular poset is a ranked poset such that for all $i$, every element of rank $i$ is covered by the same number $\alpha_{i}$ of elements and covers the same number $\beta_{i}$ of elements. For example $B_{n}$ is regular. Regular posets are known to have the LYM property.

The product (direct product, Cartesian product) of two posets $P$ and $Q$, denoted $P \times Q$, has elements $(p, q)$ for $p \in P$ and $q \in Q$ and is ordered by:

$$
(p, q) \leqslant\left(p^{\prime}, q^{\prime}\right) \Leftrightarrow p \leqslant p^{\prime} \text { in } P \text { and } q \leqslant q^{\prime} \text { in } Q \text {. }
$$

$P$ has Whitney numbers which are logarithmically concave or $L C$ if for all $j$ :

$$
\left(N_{j}(P)\right)^{2} \geqslant\left(N_{j-1}(P)\right)\left(N_{j+1}(P)\right) \text {. }
$$

LC Whitney numbers are unimodal which means that for some $r$,

$$
N_{0}(P) \leqslant N_{1}(P) \leqslant \cdots \leqslant N_{r}(P) \geqslant N_{r+1}(P) \geqslant \ldots
$$

Two ranked posets $P$ and $Q$ are compatible [17] if there exists a $d$ such that for all $i$ and $j N_{i}(P)<N_{j}(P)$ only if $N_{d-i}(Q) \leqslant N_{d-j}(Q)$. This is symmetric in $P$ and $Q$.

A chain in a ranked poset $P$ is saturated if consecutive elements in the chain are consecutive in $P$. A symmetric chain order is a ranked poset $P$ which can be partitioned into saturated chains which are symmetric about middle rank. This means that every chain contains elements of each rank from $j$ to $p-j$ for some $j$, where $p$ is the rank of $P$. Symmetric chain orders have symmetric and unimodal Whitney numbers. They have property $S$ and the Sperner property, but need not have the LYM property.

More generally, $P$ will continue to have the properties above if the symmetry condition is removed. Gansner [2] has introduced the term nested chain orders for such ranked posets. These are defined as ranked posets which can be partitioned into saturated chains which are pairwise nested, that is, of any two chains in the partition, the one containing the element of the least rank also contains the element of the greatest rank. In the language of [7], these are unimodal posets with condition $R$.

For more details and references on these notions refer to the surveys in [4] and [6].

2. $C(n, k)$ is a nested chain order. Lih proved that $C(n, k)$ has the Sperner property with a straightforward argument similar to Sperner's original theorem. (We refine this argument in §7.) Here we show by bracketing or parenthesization the considerably stronger result that $C(n, k)$ is a nested chain order. This generalizes the well-known fact that $B_{n}$ is a symmetric chain order.

THEOREM 2.1. $C(n, k)$ is a nested chain order. 
Proof. Greene and Kleitman [5] and Leeb produced an explicit symmetric chain decomposition of $B_{n}$ by bracketing: To each $S \subset X$ associate the sequence $A_{S}=$ $a_{1}, a_{2}, \ldots, a_{n}$ of 0 's and 1's, where $a_{i}=1$ if and only if $i \in S$. Whenever a 1 immediately follows a 0 in $A_{s}$, bracket or join them by placing parentheses around them. Continue this pairing procedure as long as possible by pairing an unpaired 0 with an unpaired 1 which follows it immediately, or which is separated from the 0 only by previously paired digits. For instance, for $n=11$ and $S=$ $\{1,4,6,7,8,11\}, A_{S}$ has joined pairs $3-4,5-6,2-7$, and 10-11. The bracketing is

$$
1(0(01)(01) 1) 10(01) \text {. }
$$

This bracketing is unique for all $S$, and the unpaired digits consist of 1's followed by 0's.

Sets in $B_{n}$ with the same bracketing (joined pairs) form a saturated chain which is symmetric about middle rank, $\frac{1}{2} n$. So bracketing induces a symmetric chain decomposition of $B_{n}$. In $C(n, k)$ we bracket and form chains the same way, so that $C(n, k)$ is partitioned into chains which each lie in one of the chains induced by bracketing $B_{n}$. We now consider how a chain in the bracketing of $B_{n}$ produces a chain in $C(n, k)$.

$S \in B_{n}$ belongs to $C(n, k)$ only if it intersects $Y$, that is, only if one of the first $k$ digits in $A_{S}$ is a 1 . Let $C$ be a chain in the bracketing of $B_{n}$ and let $S$ be its bottom set. First suppose $A_{S}$ contains a 1 in its first $k$ digits. Then $S \in C(n, k)$. This implies that all sets in $C$ belong to $C(n, k)$, so that $C$ is a chain induced by bracketing $C(n, k)$.

Next suppose $A_{S}$ begins with $k$ 's, and all are paired. Then this will be true all the way up the chain $C$, and no set from $C$ belongs to $C(n, k)$.

Finally suppose $A_{S}$ begins with $k$ O's, not all of which are paired. Let $T$ be the set in $C$ which covers $S . A_{T}$ is formed by changing the leftmost unpaired 0 in $A_{S}$ to a 1. Thus $A_{T}$ contains a 1 in one of the first $k$ digits, which implies that $T \in C(n, k)$. So all sets above $S$ in $C$ belong to $C(n, k)$. Deleting $S$ from $C$ produces a chain in the bracketing of $C(n, k)$.

The three cases above show that for any chain in the bracketing of $C(n, k)$ there is a $j \leqslant\left\lfloor\frac{1}{2} n\right\rfloor$ such that the chain contains sets of size $j+1, j+2, \ldots, n-j$ and possibly also a set of size $j$. It follows that these chains form a nested chain decomposition.

This proof was inspired by Gansner's study of another family of posets, the lattices of order ideals of an up-down poset [2]. For an up-down poset of $n$ points, the lattice of order ideals can be partitioned by bracketing into chains containing sets of size $j$ or $j+1$ through $n-j$. This is just like $C(n, k)$. We can also carry over Gansner's observation that each of these chains contains a set of size $\left\lceil\frac{1}{2} n\right\rceil$, so that $d_{1}(C(n, k))$ is the number of sets of size $\left\lceil\frac{1}{2} n\right\rceil$ in $C(n, k)$, as Lih already discovered. We also see that $C(n, k)$ has unimodal Whitney numbers. This result is improved in the next section: $C(n, k)$ is LC.

3. The LYM theorems. In this section we employ the theory of finite sets to obtain an even shorter proof that $C(n, k)$ has the Sperner property. Indeed, we obtain the stronger result that $C(n, k)$ is LYM and LC. This result is generalized to 
a wide class of collections of subsets. Theorem 3.3 displays a related but different class of collections of subsets which are LYM and LC.

We require the product theorem for LYM posets discovered by Harper and later by Hsieh and Kleitman. Although the product of two LYM posets $P$ and $Q$ may not be LYM, it will be true if $P$ and $Q$ are LC also.

THEOREM 3.1 [9], [10]. Suppose posets $P$ and $Q$ are $L Y M$ and LC. Then $P \times Q$ is $L Y M$ and $L C$.

One can see that $C(n, k)$ is LYM and LC as follows: $C(n, k)$ is the product of two posets. One consists of all nonempty subsets of $Y$, and the other consists of all subsets of $X-Y$. It is easy to verify that both of these posets are LYM and LC, so that by the LYM product theorem, $C(n, k)$ is itself LYM and LC.

What is happening here is that $X$ is partitioned into 2 parts, $Y$ and $X-Y$, and the posets containing the nonempty subsets of $Y$ and the subsets of $X-Y$ are LYM and LC. This suggests investigating what happens when $X$ is partitioned into an arbitrary number $m$ of parts $X_{1}, \ldots, X_{m}$ and when the sizes of intersections with each part $X_{i}$ are specified. Let $P$ be the poset containing all subsets of $X$ satisfying these constraints. Theorem 3.2 shows that $P$ is LYM and LC if the allowed sizes of the intersections form arithmetic progressions for each part $X_{i}$. A new proof of this theorem was discovered by Wareham [19]. $\$ 5$ contains examples showing that this is not true in general. In fact, $P$ need not be Sperner even, nor unimodal.

TheOREM 3.2. Let $X=\{1, \ldots, n\}$ be partitioned into parts $X_{1}, \ldots, X_{m}$. Let $I_{i} \subset\left\{0,1, \ldots,\left|X_{i}\right|\right\}$ be an arithmetic progression, $1 \leqslant i \leqslant m$. Let

$$
P=\left\{Z \subset X|| Z \cap X_{i} \mid \in I_{i}, 1 \leqslant i \leqslant m\right\},
$$

ordered by inclusion. Then $P$ is $L Y M$ and $L C$.

Proof. For all $i$, let $P_{i}=\left\{Z \subset X_{i}|| Z \mid \in I_{i}\right\}$. Then $P$ is isomorphic to $P_{1}$ $\times \cdots \times P_{m}$. It suffices to show that each $P_{i}$ is LYM and LC, for then repeated application of the product theorem implies that $P$ is LYM and LC.

Let $I_{i}=\left\{a_{i}, a_{i}+d_{i}, \ldots, a_{i}+N_{i} d_{i}\right\} . P_{i}$ is ranked, and rank $j$ in $P_{i}$ is just rank $a_{i}+j d_{i}$ in the Boolean algebra $B_{\left|X_{i}\right|} . B_{\left|X_{i}\right|}$ is LYM, which implies that $P_{i}$ is LYM also. That $P_{i}$ is LC follows from this simple inequality:

$$
\left(\begin{array}{c}
\left|X_{i}\right| \\
a_{i}+j d_{i}
\end{array}\right)^{2} \geqslant\left(\begin{array}{c}
\left|X_{i}\right| \\
a_{i}+(j-1) d_{i}
\end{array}\right)\left(\begin{array}{c}
\left|X_{i}\right| \\
a_{i}+(j+1) d_{i}
\end{array}\right) .
$$

It is interesting to note that this last observation that $P_{i}$ is LC can be generalized. Specifically, for any LC poset $Q$ and any arithmetic progression $I$, the poset $Q^{\prime}=\{x \in Q \mid r(x) \in I\}$ is also LC. So Theorem 3.2 can be generalized beyond Boolean algebras to products of arbitrary LYM and LC posets. Now we give some applications of Theorem 3.2.

EXAMPLE 3.1. $C(n, k)$ is $P$ in Theorem 3.2 when $m=2, X_{1}=Y=\{1, \ldots, k\}$, $X_{2}=X-Y=\{k+1, \ldots, n\}, I_{1}=\{1, \ldots, k\}$, and $I_{2}=\{0,1, \ldots, n-k\}$. Hence, $C(n, k)$ is LYM and LC. It follows that $C(n, k)$ is Sperner. 
ExAmple 3.2. With $X$ and $Y$ as above with $1 \leqslant l \leqslant k$, let $P$ consist of all subsets of $X$ which intersect $Y$ at least $l$ times. This is like Example 3.1, but now $I_{1}=\{l, \ldots, k\}$, which is more general. By the theorem, $P$ is LYM and LC. Here are two expressions for $N_{j}(P), 0 \leqslant j \leqslant n-l$;

$$
\begin{aligned}
N_{j}(P) & =\left(\begin{array}{c}
n \\
j+l
\end{array}\right)-\sum_{i=0}^{l-1}\left(\begin{array}{c}
k \\
i
\end{array}\right)\left(\begin{array}{c}
n-k \\
j+l-i
\end{array}\right) \\
& =\sum_{i=0}^{j}\left(\begin{array}{c}
k \\
l+i
\end{array}\right)\left(\begin{array}{c}
n-k \\
j-i
\end{array}\right) .
\end{aligned}
$$

$d_{1}(P)$ is the largest $N_{j}(P)$. For $l=1$, this is attained by $j=\left\lceil\frac{1}{2} n\right\rceil-1$, independent of $k$. But in general a formula for $j$ maximizing $N_{j}(P)$ will depend on $k$. For example, if $n=8$, and $l=2, N_{j}(P)$ is largest when $j=3$ if $k=3$ and when $j=2$ if $k=4$. See $\$ 8$ for more on this poset.

EXAMPLE 3.3. $m$-color $X$ and let $P$ consist of all subsets of $X$ containing at least $a_{i}$ and at most $b_{i}$ elements of color $i$. Then $P$ is LYM and LC.

EXAMPLE 3.4. Let $P$ consist of all subsets of $X$ containing an even number of odd elements and an odd number of even elements. That is, $X_{1}=I_{2}=\{1,3, \ldots\}$ and $X_{2}=I_{1}=\{0,2, \ldots\}$. Then $I_{1}$ and $I_{2}$ are arithmetic progressions, so $P$ is LYM and $\mathrm{LC}$.

ExAmple 3.5. Let $n=4, m=2, X_{1}=\{1,2\}, X_{2}=\{3,4\}, I_{1}=\{0,1,2\}$, and $I_{2}=\{0,2\}$. This shows that the ranks in $P$ do not correspond to the collections of sets in $P$ of the same size. Here $\{3,4\}$ has rank 1 and $\{1,2\}$ has rank 2. Nevertheless, this $P$ is LYM and LC by the theorem. (This $P$ is isomorphic to $C(3,3)$, i.e., to $B_{3}$ with $\varnothing$ deleted.)

Why do we require the condition that $I_{i}$ be an arithmetic progression in Theorem 3.2? Although this condition is not necessarry, we do need something. Consider what happens when no conditions are placed on the $I_{i}$. The posets $P_{i}$ remain ranked and still inherit the LYM property from the Boolean algebra of all subsets of $X_{i}$. However, the Whitney numbers of $P_{i}$ are merely unimodal and are not always LC as the product theorem requires. The smallest example of this is with $\left|X_{i}\right|=8$ and $I_{i}=\{0,1,4\} . P_{i}$ has Whitney numbers $1,8,70$ in this case.

Indeed $P$ is not LYM nor LC for general $I_{i}$. In $\$ 5$ examples are given to demonstrate this. Even the Sperner property fails in general, although it holds under certain conditions not covered by Theorem 3.2. $\$ 4$ presents these results.

For the remainder of this section we will show that a different, but related, class of posets has the LYM property. The motivating example is this problem: With $X$ and $Y$ as usual, let $P$ be the subsets of $X$ with an equal number of elements inside and outside $Y$. What can we say about $P$ this time? As in Theorem 3.2 we can show that $P$ is LYM and LC, and again we can prove a more general result for arbitrary partitions of $X$ with restrictions on the sets $I_{i}$.

THEOREM 3.3. Let $X=\{1, \ldots, n\}$ be partitioned into $m$ parts, $X_{1}, \ldots, X_{m}$. For $1 \leqslant i \leqslant m$ let $I_{i} \subset\{0,1, \ldots\}$ be an arithmetic progression of integers of length 


$$
\begin{aligned}
& N+1, \text { say } I_{i}=\left\{a_{i}, a_{i}+d_{i}, \ldots, a_{i}+N d_{i}\right\}, \text { where } d_{i}>0 . \text { Let } \\
& \quad P=\left\{Z \subset X \mid(\exists \lambda \in\{0,1, \ldots, N\})\left(\left|Z \cap X_{i}\right|=a_{i}+\lambda d_{i}, 1<i<m\right)\right\} .
\end{aligned}
$$

Then $P$ is $L Y M$ and $L C$.

Proof. Each element of rank $\lambda$ in $P$ is the union over $i$ of a subset of $X_{i}$ of size $a_{i}+\lambda d_{i}$. It follows by symmetry that $P$ is regular, hence LYM. $P$ has Whitney numbers $N_{\lambda}(P)=\left(N_{\lambda}\left(P_{1}\right)\right) \cdots\left(N_{\lambda}\left(P_{m}\right)\right)$, where $P_{i}=\left\{Z \subset X_{i}|| Z \mid \in I_{i}\right\}$. From the proof of Theorem 3.2, $P_{i}$ is LC. The product of nonnegative LC sequences is LC so $P$ is LC.

EXAMPLE 3.6. With $X$ and $Y$ as usual consider

$$
P=\{Z \subset X|| Z \cap Y|=| Z \cap(X-Y) \mid\} .
$$

$P$ is LYM and LC by Theorem 3.3 with $X_{1}=Y, X_{2}=X-Y, I_{1}=I_{2}=$ $\{0,1, \ldots\}$.

EXAMPLE 3.7. More generally, given numbers $a_{i}, \ldots, a_{m}$, let

$$
P=\left\{Z \subset X\left|a_{1}\right| Z \cap X_{1}\left|=\cdots=a_{m}\right| Z \cap X_{m} \mid\right\} .
$$

Then by Theorem 3.3 $P$ is LYM and LC.

In Theorem 3.2 the numbers $\left|Z \cap X_{i}\right|$ are selected independently for each $i$ (in "series"). In 3.3 they are picked for all $i$ simultaneously (in "parallel"). 3.2 is deeper and more interesting because it produces LYM posets that are not regular.

4. Two more approaches. There are two more product theorems which we apply in this section to obtain sufficient conditions on the sets $I_{i}$ of Theorem 3.2 to guarantee that the poset $P$ defined there has the Sperner property.

Here is the product theorem for symmetric chain orders (see [6] for a proof).

TheOREM 4.1. If $P$ and $Q$ are symmetric chain orders, then so is $P \times Q$.

We say a subset $S$ of $\{0,1, \ldots, r\}$ is symmetric if for all $j, j \in S$ if and only if $r-j \in S$. Here is a theorem which gives sufficient conditions for $P$ to be a symmetric chain order. Recall that symmetric chain orders have property $S$ (and, hence, the Sperner property) and unimodal Whitney numbers.

THEOREM 4.2. Let $X=\{1, \ldots, n\}$ be partitioned into parts $X_{1}, \ldots, X_{m}$. Let $I_{i}$ be symmetric in $\left\{0,1, \ldots,\left|X_{i}\right|\right\}, 1 \leqslant i \leqslant m$. Then

$$
P=\left\{Z \subset X|| Z \cap X_{i} \mid \in I_{i}, 1 \leqslant i \leqslant m\right\}
$$

is a symmetric chain order.

Proof. The poset of all subsets of $X_{i}$ is isomorphic to $B_{\left|X_{i}\right|}$, a symmetric chain order. The symmetric chains, restricted to $P_{i}=\left\{Z \subset X_{i}|| Z \mid \in I_{i}\right\}$, establish that $P_{i}$ is a symmetric chain order. By repeated application of Theorem 4.1, $P$ is a symmetric chain order, since $P$ is isomorphic to $P_{1} \times \cdots \times P_{m}$.

For partitions into just two parts there is another approach we can take to get sufficient conditions for $\boldsymbol{P}$ to have the Sperner property. This is to use this product theorem for posets with property $S$. 
THEOREM 4.3 [17]. Let $P$ and $Q$ be unimodal posets with property $S$. If $P$ and $Q$ are compatible, then $P \times Q$ is unimodal and Sperner.

TheOREM 4.4. Suppose $X=\{1, \ldots, n\}$ is partitioned into two parts, $X_{1}$ and $X_{2}$. For $i=1,2$ let $I_{i} \subset\left\{0,1, \ldots,\left|X_{i}\right|\right\}$, and let $P_{i}=\left\{Z \subset X_{i}|| Z \mid \in I_{i}\right\}$. Let

$$
P=\left\{Z \subset X|| Z \cap X_{i} \mid \in I_{i}, i=1,2\right\} .
$$

If $P_{1}$ and $P_{2}$ are compatible, then $P$ is unimodal and Sperner.

Proof. As in the proof of Theorem 3.2, $P_{1}$ and $P_{2}$ are LYM. Hence they have property $S$. Their Whitney numbers are subsequences of the Whitney numbers for $B_{\left|X_{1}\right|}$ and $B_{\left|X_{2}\right|}$, so are unimodal. By hypothesis $P_{1}$ and $P_{2}$ are compatible. Hence by Theorem 4.3, $P=P_{1} \times P_{2}$ is unimodal and Sperner.

Unfortunately, Theorem 4.3 does not permit us to say anything about partitions of $X$ into more than two parts, because it only implies that $P_{1} \times P_{2}$ is Sperner and we require that $P_{1} \times P_{2}$ has property $S$ to use the theorem on $P_{1} \times P_{2} \times P_{3}$. An application of Theorem 4.4 is included in Example 5.2.

Another instance where Theorem 4.4 may be applied is to $C(n, k)$. Simply take $X_{1}, X_{2}, I_{1}$, and $I_{2}$ as in Example 3.1.

5. Counterexamples. This section contains counterexamples to several plausible generalizations of the results in the last two sections.

Example 3.2, viewed in a different light, says that if we let $\mathcal{S}$ be the collection of subsets of $Y$ of size $l$, then the poset

$$
P_{\delta}=\{Z \subset X \mid(\exists W \in \delta)(W \subset Z)\},
$$

i.e., the filter generated by $\mathcal{S}$, is LYM. We show now that for arbitrary collections $\delta$ of $l$-subsets of $X, P_{\delta}$ is not even Sperner. This supplies a counterexample to Lih's conjecture [14] that in any Sperner poset the filter generated by a collection of elements of fixed rank is Sperner.

EXAMPLE 5.1. Let $n=6$ and let

$$
\delta=\{1234,1256,1356,1456,2356,2456,3456\}
$$

brackets omitted. Then $P_{\delta}$ has rank 2 and Whitney numbers 7, 6, 1, starting with $N_{0} . P_{\delta}$ is not Sperner because there is an antichain of size 8,

$$
\{12345,12346,1256,1356,1456,2356,2456,3456\} \text {. }
$$

In the remaining example we follow the notation and proof of Theorem 3.2 except that the sets $I_{i}$ are not restricted to arithmetic progressions.

EXAMPLE 5.2. Here is a small example showing that $P$ is not LYM in general. Take $n=10, X_{1}=\{1, \ldots, 9\}, X_{2}=\{10\}, I_{1}=\{0,1,4\}$, and $I_{2}=\{0,1\} . P$ has Whitney numbers $1,10,135,126$. Here is an antichain in $P$ which violates the LYM inequality,

$$
\{\{10\}\} \cup\left\{Z \subset X_{1}|| Z \mid=4\right\} .
$$

However, Theorem 4.4 tells us that $P$ is Sperner because $P_{1}$ and $P_{2}$ are compatible with $d=2: P_{1}$ has Whitney numbers $1,9,126, P_{2}$ has Whitney numbers $1,1 . P$ actually has property $S$. 
EXAMPLE 5.3. Now we show that $P$ is not unimodal in general. If there are examples with small $n$, say $n \leqslant 20$, they are probably rather complicated. Here we go to a large $n, n=400$, but get a simple example. Let $m=2, X_{1}=\{1, \ldots, 200\}$, $X_{2}=\{201, \ldots, 400\}$, and $I_{1}=I_{2}=\{100,109,110\} . P_{1}$ and $P_{2}$ are isomorphic rank 2 posets. It is convenient to define

$$
\alpha=\frac{\left(\begin{array}{l}
200 \\
109
\end{array}\right)}{\left(\begin{array}{l}
200 \\
100
\end{array}\right)} \approx .446 ; \quad \beta=\frac{\left(\begin{array}{l}
200 \\
110
\end{array}\right)}{\left(\begin{array}{l}
200 \\
100
\end{array}\right)} \approx .369 .
$$

$P$ has rank 4 and Whitney numbers as follows.

$$
\begin{aligned}
& N_{0}(P)=\left(\begin{array}{l}
200 \\
100
\end{array}\right)^{2}, \\
& N_{1}(P)=2 \alpha N_{0}(P) \approx(.892) N_{0}(P), \\
& N_{2}(P)=\left(2 \beta+\alpha^{2}\right) N_{0}(P) \approx(.938) N_{0}(P), \\
& N_{3}(P)=2 \alpha \beta N_{0}(P) \approx(.329) N_{0}(P), \\
& N_{4}(P)=\beta^{2} N_{0}(P) \approx(.136) N_{0}(P) .
\end{aligned}
$$

$N_{1}(P)$ is less than $N_{0}(P)$ and $N_{2}(P)$, so $P$ is not unimodal. None of our theorems apply to $P$, but nonetheless it can be shown that $P$ has property $S$. (To do this, use the result [3] that every finite poset has a $k$-family of maximum size made up of full orbits under the automorphism group of the poset. In this example there is only one orbit at each rank, except rank 2, which has two orbits. That $P$ has property $S$ follows easily.) In fact, a careful study shows that $P$ has the LYM property.

EXAMPLE 5.4. Once a poset $P$ which is not unimodal was constructed, it appeared likely that posets $P$ without the Sperner property would be lurking nearby. Fortunately this is the case. Although $P$ in Example 5.3 is Sperner (and even LYM), we need only take its product with $B_{1}$ to lose the Sperner property. Construct this poset, call it $P^{\prime}$, as follows: Take $n=401, m=3, X_{1}, X_{2}, I_{1}$, and $I_{2}$ as in Example 5.3, $X_{3}=\{401\}$, and $I_{3}=\{0,1\}$. By design $N_{j}\left(P^{\prime}\right)=N_{j}(P)+$ $N_{j-1}(P), 0 \leqslant j \leqslant 5$. Hence,

$$
\begin{aligned}
& N_{0}\left(P^{\prime}\right)=N_{0}(P)=\left(\begin{array}{l}
200 \\
100
\end{array}\right)^{2}, \\
& N_{1}\left(P^{\prime}\right) \approx(1.892) N_{0}(P), \\
& N_{2}\left(P^{\prime}\right) \approx(1.830) N_{0}(P), \\
& N_{3}\left(P^{\prime}\right) \approx(1.267) N_{0}(P), \\
& N_{4}\left(P^{\prime}\right) \approx(.465) N_{0}(P), \\
& N_{5}\left(P^{\prime}\right) \approx(.136) N_{0}(P) .
\end{aligned}
$$

So $P^{\prime}$ is back to being unimodal, but here is an antichain larger than any of the $N_{j}\left(P^{\prime}\right)$. 


$$
\begin{aligned}
A= & \left\{Z \subset X|| Z \cap X_{1}|=| Z \cap X_{2}|=100,| Z \cap X_{3} \mid=1\right\} \\
& \cup\left\{Z \subset X|| Z \cap X_{1}|=| Z \cap X_{2}|=109,| Z \cap X_{3} \mid=0\right\} \\
& \cup\left\{Z \subset X|| Z \cap X_{1}|=110,| Z \cap X_{2}|=100,| Z \cap X_{3} \mid=0\right\} \\
& \cup\left\{Z \subset X|| Z \cap X_{1}|=100,| Z \cap X_{2}|=110,| Z \cap X_{3} \mid=0\right\},
\end{aligned}
$$

i.e., take sets in rank 2 of $P$, together with sets in rank 0 of $P$ with 401 added.

$$
|A|=N_{0}(P)+N_{2}(P) \approx(1.938) N_{0}(P),
$$

which exceeds the largest $N_{j}\left(P^{\prime}\right)$.

One might hope that Theorem 4.1, the product theorem for symmetric chsin orders, could be extended to nested chain orders. Examples 5.3 and 5.4 show that this is not possible. For, each poset $P_{i}$ consists of full ranks from $B_{\left|X_{i}\right|}$, so the symmetric chain decomposition of $B_{\left|X_{i}\right|}$ induces a nested chain decomposition of $P_{i}$. In Example 5.3, $P=P_{1} \times P_{2}$ is not unimodal, so cannot be a nested chain order. In Example 5.4, $P=P_{1} \times P_{2} \times P_{3}$ is not even Sperner.

6. $C(n, k)$ is LYM via the Kruskal-Katona Theorem. A poset $\boldsymbol{P}$ has the LYM property if and only if it has the normalized matching property, which is that for every rank $j$ and every set $\delta$ of elements of rank $j$,

$$
\frac{|\delta *|}{N_{j+1}(P)} \geqslant \frac{|\delta|}{N_{j}(P)},
$$

where $\mathcal{S}^{*}$ denotes the set of elements which cover some element of $\mathcal{S}$ [13].

It follows that $C(n, k)$ can (again) be shown to be LYM by proving that equation (2) below holds for all $m$ and for all collections $\delta$ of $m$-subsets in $C(n, k)$. Such a collection $\mathcal{S}$ also lies in the Boolean algebra of all subsets of $X, B_{n}$. The subsets $\mathcal{S}^{*}$ which cover $\mathcal{S}$ are the same in $B_{n}$ as in $C(n, k)$. We already know that $B_{n}$ is LYM, so taking $P=B_{n}$ in (1) implies that

$$
\frac{\left|\mathcal{S}^{*}\right|}{|\mathcal{S}|} \geqslant \frac{\left(\begin{array}{c}
n \\
m+1
\end{array}\right)}{\left(\begin{array}{c}
n \\
m
\end{array}\right)}=\frac{n-m}{m+1} .
$$

However, this bound on $\left|\delta^{*}\right| /|\delta|$ is weaker than that required by (2).

The Kruskal-Katona theorem [11], [14] provides the best-possible lower bound on $|\mathcal{S} *| / \mathcal{S} \mid$ for collections $\mathcal{S}$ with $|\mathcal{S}|$ given. Specifically, suppose

$$
|\delta|=\left(\begin{array}{c}
a_{n-m} \\
n-m
\end{array}\right)+\left(\begin{array}{c}
a_{n-m-1} \\
n-m-1
\end{array}\right)+\cdots+\left(\begin{array}{c}
a_{i} \\
i
\end{array}\right)
$$

where $a_{n-m}>a_{n-m-1}>\cdots>a_{i} \geqslant i>0$. (Such $a$ 's exist and are unique.) Then

$$
\left|\mathcal{S}^{*}\right| \geqslant\left(\begin{array}{c}
a_{n-m} \\
n-m-1
\end{array}\right)+\left(\begin{array}{c}
a_{n-m-1} \\
n-m-2
\end{array}\right)+\cdots+\left(\begin{array}{c}
a_{i} \\
i-1
\end{array}\right) .
$$

(This is the complement of Theorem 8.1 in [4].) Although this best-possible bound on $\left|\mathcal{S}^{*}\right|$ for given $|\delta|$ is stronger than that in (2), it is not suitable for proving (2). We require an induction argument. However, we use another version of the Kruskal-Katona theorem to obtain collections $\delta$ for which $\left|\delta^{*}\right|$ attains its lower bound. 
The new bound on $|\mathcal{E}| /|\mathcal{S}|$ given in (2) actually works for $\mathcal{S}$ in $B_{n}$, not just in $C(n, k)$. So Theorem 6.1 is stated for $B_{n}$. Unfortunately, it does not appear that this approach can be extended to more general problems, such as the posets discussed in Example 3.2 and again in $\$ 8$.

THEOREM 6.1. Let $\delta$ be a collection of $m$-subsets of $X=\{1, \ldots, n\}$ with $1 \leqslant|\delta| \leqslant\left(\begin{array}{c}n \\ m\end{array}\right)-\left(\begin{array}{c}n-k \\ m\end{array}\right)$. Then

$$
\frac{\left|\mathcal{S}^{*}\right|}{|\mathcal{S}|} \geqslant \frac{\left(\begin{array}{c}
n \\
m+1
\end{array}\right)-\left(\begin{array}{c}
n-k \\
m+1
\end{array}\right)}{\left(\begin{array}{c}
n \\
m
\end{array}\right)-\left(\begin{array}{c}
n-k \\
m
\end{array}\right)} .
$$

Proof. According to the Kruskal-Katona theorem [11], [14] $|\delta *|$ is minimized over all collections $\delta$ of $m$-subsets of $X$, with $|\delta|$ equal to some fixed value $s$, by taking the first $s m$-subsets of $X$ under reverse lexicographic ordering. (This is the complement of $[4$, p. 64].) This means that each $m$-subset $Z$ of $X$ is identified with an $n$-digit binary number $a_{1}, a_{2}, \ldots, a_{n}$ by $a_{i}=1$ if $i \in Z$ and $a_{i}=0$ if $i \notin Z$. To minimize $\left|\delta^{*}\right|$ take $\delta$ to be $\delta(n, m, s)$, which we define to be the $m$-subsets of $X$ corresponding to the $s$ largest binary numbers with $m$ ones and length $n$.

Let $\varphi(n, m, s)=\left|\delta^{*}(n, m, s)\right| / s$, where $\delta^{*}(n, m, s)$ means $(\delta(n, m, s))^{*}$. So $\varphi(n, m, s)$ is the minimum of $|\mathcal{} *| /|\mathcal{S}|$ for all $\delta$ with $|\mathcal{S}|=s$. The theorem thus reduces to showing that

$$
\varphi(n, m, s) \geqslant \frac{\left(\begin{array}{c}
n \\
m+1
\end{array}\right)-\left(\begin{array}{c}
n-k \\
m+1
\end{array}\right)}{\left(\begin{array}{c}
n \\
m
\end{array}\right)-\left(\begin{array}{c}
n-k \\
m
\end{array}\right)},
$$

for $1 \leqslant s \leqslant\left(\begin{array}{c}n \\ m\end{array}\right)-\left(\begin{array}{c}n-k \\ m\end{array}\right)$.

Let

$$
e(n, m, i)=\left(\begin{array}{c}
n \\
m
\end{array}\right)-\left(\begin{array}{c}
n-i \\
m
\end{array}\right), \quad 0 \leqslant i \leqslant n .
$$

This is the number of $m$-subsets of $X$ which intersect $\{1, \ldots, i\}$. When $s=$ $e(n, m, k), \delta(n, m, s)$ consists of all $m$-subsets of $X$ which intersect $Y=$ $\{1, \ldots, k\}$, that is, $\delta(n, m, s)$ contains all $m$-sets in $C(n, k)$. So $\mathcal{S}^{*}(n, m, s)$ is all $(m+1)$-sets in $C(n, k)$ for this $s$. Hence the right side of (3) is just $\varphi(n, m, e(n, m, k))$, and (3) is equivalent to

$$
\varphi(n, m, s) \geqslant \varphi(n, m, e(n, m, k)), \quad 1 \leqslant s \leqslant e(n, m, k) .
$$

We prove (4) for all $m$ and $k$ by induction on $n$. For $n=1$, (4) is trivial, so assume $n>1$. The proof might not be so hard if $\varphi(n, m, s) \geqslant \varphi(n, m, s+1)$ for all $s$, but this is not true in general. However, (4) follows from these two observations, (5) and (6), which are proved below,

$$
\varphi(n, m, e(n, m, i)) \geqslant \varphi(n, m, e(n, m, i+1)),
$$

and, for $e(n, m, i)<s<e(n, m, i+1)$,

$$
\varphi(n, m, s) \geqslant \varphi(n, m, e(n, m, i+1)) .
$$

This trivial lemma is used repeatedly in the proof. 
Lemma. Let $A, B, C, D \geqslant 0, B, D \neq 0$, such that $A / B \geqslant C / D$. Then,

$$
\frac{A}{B} \geqslant \frac{A+C}{B+D} \geqslant \frac{C}{D} \text {. }
$$

Proof of (5). For any $j$, the range $e(n, m, j)<s \leqslant e(n, m, j+1)$ includes $\alpha_{j}$ values of $s$ where

$$
\alpha_{j}=e(n, m, j+1)-e(n, m, j)=\left(\begin{array}{c}
n-j-1 \\
m-1
\end{array}\right) .
$$

In reverse lexicographic ordering the $s$ th largest $m$-sets in $B_{n}$ with $s$ in this range correspond to the $m$-subsets of $X$ which are disjoint from $\{1, \ldots, j\}$ and which contain $j+1$. So the $(m+1)$-sets in $B_{n}$ which cover $m$-sets in this range but cover none of the first $e(n, m, j) m$-sets are precisely the $(m+1)$-sets which contain $j+1$ and are disjoint from $\{1, \ldots, j\}$. The number $\beta_{j}$ of such $(m+1)$-sets is

$$
\beta_{j}=e(n, m+1, j+1)-e(n, m+1, j)=\left(\begin{array}{c}
n-j-1 \\
m
\end{array}\right) .
$$

In the range $1 \leqslant s \leqslant e(n, m, i)$ there are $\alpha_{0}+\cdots+\alpha_{i-1} m$-sets and the number of $(m+1)$-sets which cover them is $\beta_{0}+\cdots+\beta_{i-1}$. So (5) is equivalent to

$$
\frac{\beta_{0}+\cdots+\beta_{i-1}}{\alpha_{0}+\cdots+\alpha_{i-1}} \geqslant \frac{\beta_{0}+\cdots+\beta_{i}}{\alpha_{0}+\cdots+\alpha_{i}},
$$

which follows from the lemma, applied repeatedly, together with the observation that

$$
\frac{\beta_{j}}{\alpha_{j}}=\frac{n-m-j}{m}
$$

decreases as $j$ increases.

Proof of (6). Fix $s, e(n, m, i)<s<e(n, m, i+1)$. Let $\gamma_{1}=e(n, m, i), \gamma_{2}=s$ $-e(n, m, i)$, and $\gamma_{3}=e(n, m, i+1)-s$. Let $\delta_{1}$ be the number of $(m+1)$-sets covering the first $\gamma_{1} m$-sets in $B_{n}$. So $\delta_{1}=e(n, m+1, i)$. Let $\delta_{2}$ be the number of $(m+1)$-sets covering the next $\gamma_{2} m$-sets but which do not cover any of the first $\gamma_{1}$ $m$-sets. Similarly $\delta_{3}$ is the number of new $(m+1)$-sets covering the next $\gamma_{3} m$-sets.

(6) is equivalent to this inequality, which it remains to prove,

$$
\frac{\delta_{1}+\delta_{2}}{\gamma_{1}+\gamma_{2}} \geqslant \frac{\delta_{1}+\delta_{2}+\delta_{3}}{\gamma_{1}+\gamma_{2}+\gamma_{3}}
$$

For $t$ satisfying $e(n, m, i)<t \leqslant e(n, m, i+1)$, let $T$ be the $t$ th $m$-set and let $T^{*}$ be any $(m+1)$-set which covers $T$ and which covers none of the first $t-1 m$-sets. $T$ and $T^{*}$ both contain $i+1$ and are disjoint from $\{1, \ldots, i\}$. Throwing out the first $i+1$ elements of $X$, this is the same as taking an $(m-1)$-subset of $\{i+2, \ldots, n\}$ and an $m$-set in $\{i+2, \ldots, n\}$ which contains it. Hence,

$$
\frac{\delta_{2}}{\gamma_{2}}=\varphi\left(n-i-1, m-1, \gamma_{2}\right) \text {. }
$$

By induction on $n$, we may apply (4) to obtain

$$
\varphi\left(n-i-1, m-1, \gamma_{2}\right) \geqslant \varphi(n-i-1, m-1, e(n-i-1, m-1, n-i-1)) \text {. }
$$


The last expression here corresponds to taking all $\gamma_{2}+\gamma_{3} m$-sets in the range in $t$, so that

$$
\frac{\delta_{2}+\delta_{3}}{\gamma_{2}+\gamma_{3}}=\varphi(n-i-1, m-1, e(n-i-1, m-1, n-i-1)) .
$$

The last three expressions lead to

$$
\frac{\delta_{2}}{\gamma_{2}} \geqslant \frac{\delta_{2}+\delta_{3}}{\gamma_{2}+\gamma_{3}} .
$$

If $\gamma_{1}=0$, that is, if $i=0$, (7) follows from (8) by adding $\delta_{1} / \gamma_{2}$ to the left side of (8) and $\delta_{1} /\left(\gamma_{2}+\gamma_{3}\right)$ to the right side. Henceforth assume $\gamma_{1}>0$. The proof of (7) is done in two cases.

Case 1. Suppose $\delta_{1} / \gamma_{1} \geqslant \delta_{2} / \gamma_{2}$. By the lemma,

$$
\frac{\delta_{1}+\delta_{2}}{\gamma_{1}+\gamma_{2}} \geqslant \frac{\delta_{2}}{\gamma_{2}} \text {. }
$$

By the lemma and (8), $\delta_{2} / \gamma_{2} \geqslant \delta_{3} / \gamma_{3}$. Hence,

$$
\frac{\delta_{1}+\delta_{2}}{\gamma_{1}+\gamma_{2}} \geqslant \frac{\delta_{3}}{\gamma_{3}}
$$

which implies (7), by the lemma.

Case 2. Suppose $\delta_{1} / \gamma_{1} \leqslant \delta_{2} / \gamma_{2}$. By the lemma,

$$
\frac{\delta_{1}+\delta_{2}}{\gamma_{1}+\gamma_{2}} \geqslant \frac{\delta_{1}}{\gamma_{1}}
$$

We already proved (5) so we may use it here. In this notation, it states that

$$
\frac{\delta_{1}}{\gamma_{1}} \geqslant \frac{\delta_{1}+\delta_{2}+\delta_{3}}{\gamma_{1}+\gamma_{2}+\gamma_{3}}
$$

These last two inequalities combine to give (7).

As a consequence of (5), the best bound on $\left|\delta^{*}\right| /|\delta|$ that can be obtained from Theorem 6.1 is by taking the smallest $k$ in (2) such that $|\mathcal{S}|<\left(\begin{array}{c}n \\ m\end{array}\right)-\left(\begin{array}{c}n-k \\ m\end{array}\right)$. When $|\delta|=\left(\begin{array}{c}n \\ m\end{array}\right)-\left(\begin{array}{c}n-k \\ m\end{array}\right)$ the bound in (2) is best-possible.

7. Finding all maximum-sized antichains in $C(n, k)$. Sperner [18] showed that in any maximum-sized antichain $A$ in $B_{n}$, all sets in $A$ have the same size. We show this holds more generally in $C(n, k)$ and we find all of these antichains.

In [6] Sperner's result was generalized by characterizing all regular posets $P$ with this "strong Sperner property" that in every maximum-sized antichain in $P$ all elements have the same rank. This does not apply here because $C(n, k)$ is not quite regular: although every $m$-set in $C(n, k)$ is covered by $n-m(m+1)$-sets, the number of $(m-1)$-sets covered by an $m$-set $Z$ is $m$ if $|Z \cap Y|>1$ and $m-1$ if $|Z \cap Y|=1$. That $C(n, k)$ is LYM implies that every element of every maximumsized antichain belongs to a maximum-sized rank, but this is not as strong as the desired result. LYM posets can contain maximum-sized antichains containing elements from more than one of the maximum-sized ranks. So instead we have to 
start over, and we make no use of the fact that $C(n, k)$ is LYM. We generalize Sperner's proof for $B_{n}$. Lih's proof that $C(n, k)$ is Sperner and

$$
d_{1}(C(n, k))=\left(\begin{array}{c}
n \\
\left\lceil\frac{1}{2} n\right\rceil
\end{array}\right)-\left(\begin{array}{c}
n-k \\
\left\lceil\frac{1}{2} n\right\rceil
\end{array}\right)
$$

is similar. We are merely being more careful.

$C_{m}$ denotes the collection of sets in $C(n, k)$ of size $m$, i.e., of rank $m-1$.

TheOREM 7.1. $C(n, k)$ is Sperner,

$$
d_{1}(C(n, k))=\left(\begin{array}{c}
n \\
\left\lceil\frac{1}{2} n\right\rceil
\end{array}\right)-\left(\begin{array}{c}
n-k \\
\left\lceil\frac{1}{2} n\right\rceil
\end{array}\right)
$$

and the maximum-sized antichains in $C(n, k)$ are

1. $C\left\lceil\frac{1}{2} n\right\rceil$,

2. $C_{\frac{1}{2}(n-1)}$, for odd $n$ and $i \geqslant \frac{1}{2}(n+3)$, and

3. $C_{\frac{1}{2}(n+2)}$, for even $n$ and $k=1$.

Proof. Consider the bipartite graph $G_{m}$ with vertices in $C_{m} \cup C_{m+1}$ and edges between $Z \in C_{m}$ and $Z^{*} \in C_{m+1}$ if $Z \subset Z^{*}$. This observation is simple to check:

Fact $1 . G_{m}$ is connected.

For a collection $S$ of sets of the same size in $C(n, k), S^{*}$ (respectively, $S_{*}$ ) denotes the collection of sets in $C(n, k)$ which cover (respectively, are covered by) sets in $S$.

Let $S \subset C_{m}$ and consider the graph $G^{S}$ with vertices in $S \cup S^{*}$ and edges in $G_{m}$. Each set $Z \in S$ is covered by $n-m$ sets in $S^{*}$, so $G^{S}$ has $(n-m)|S|$ edges. On the other hand, a set $Z^{*} \in S^{*}$ covers $m+1$ sets in $C_{m}$, unless $\left|Z^{*} \cap Y\right|=1$, when $Z^{*}$ covers just $m$ sets. These sets covered by $Z^{*}$ need not all belong to $S$. Adding over $Z^{*} \in S^{*}$, the number of edges in $G_{m}$ is at most $(m+1)\left|S^{*}\right|$. This implies

Fact 2. $(n-m)|S| \leqslant(m+1)\left|S^{*}\right|$ for $S \subset C_{m}$.

Similarly we can count edges in the graph $G_{S}$ induced by $G_{m}$ on the vertices $S \cup S_{*}$. Each $Z \in S$ covers $m-1$ or $m$ sets, so $G_{S}$ has at least $(m-1)|S|$ edges. Each set $Z_{*} \in S_{*}$ is covered by $n-m+1$ sets in $C_{m}$, so $G_{S}$ has $(n-m+1)\left|S_{*}\right|$ edges in all. Hence we have

Fact 3. $(m-1)|S| \leqslant(n-m+1)\left|S_{*}\right|$, for $S \subset C_{m}$.

Let $A$ be a maximum-sized antichain in $C(n, k)$, and let $A_{m}=A \cap C_{m}$ for all $m$. We have three cases to consider.

Case 1. Suppose for some $m \leqslant\left\lceil\frac{1}{2} n\right\rceil-1$ that $A_{m} \neq \varnothing$ and $A_{0}=A_{1}=\cdots=$ $A_{m-1}=\varnothing$. Then $m+1 \leqslant n-m$, so that by Fact 2 with $S=A_{m},|S|<\left|S^{*}\right|$, and $|S|=\left|S^{*}\right|$ only if $n-m=m+1$. If $S$ is replaced by $S^{*}$ in $A$, we get another antichain $A^{\prime}$ with $\left|A^{\prime}\right| \geqslant|A|$. But $A$ is maximum-sized, so $\left|A^{\prime}\right|<|A|$. Hence,

$$
\begin{aligned}
\left|A^{\prime}\right|=|A| & \Rightarrow|S|=\left|S^{*}\right| \\
& \Rightarrow n-m=m+1 \\
& \Rightarrow m=\frac{1}{2}(n-1) .
\end{aligned}
$$


This forces $n$ to be odd. Further, $(n-m)|S|=(m+1)\left|S^{*}\right|$ means that every set covered by $S^{*}$ lies in $S$ (by looking closely at the proof of Fact 2). This means that in $G_{m}$, no edge joins a set in $S \cup S^{*}$ with a set not in $S \cup S^{*}$. But $G_{m}$ is connected (Fact 1). Hence $S=C_{m}$ and $S^{*}=C_{m+1}$, with $m=\frac{1}{2}(n-1)$,

$$
\begin{array}{r}
\Rightarrow|S|=\left(\begin{array}{c}
n \\
\frac{1}{2}(n-1)
\end{array}\right)-\left(\begin{array}{c}
n-k \\
\frac{1}{2}(n-1)
\end{array}\right) \\
\text { and }\left|S^{*}\right|=\left(\begin{array}{c}
n \\
\frac{1}{2}(n+1)
\end{array}\right)-\left(\begin{array}{c}
n-k \\
\frac{1}{2}(n+1)
\end{array}\right) .
\end{array}
$$

Now $|S|=\left|S^{*}\right|$ forces

$$
\left(\begin{array}{c}
n-k \\
\frac{1}{2}(n-1)
\end{array}\right)=\left(\begin{array}{c}
n-k \\
\frac{1}{2}(n+1)
\end{array}\right)
$$

Since $k \geqslant 1$, this is true only if both of these coefficients are zero, which is true when $k \geqslant \frac{1}{2}(n+3)$. So our original assumption that $m \leqslant\left\lceil\frac{1}{2} n\right\rceil-1$ works only if $n$ is odd, $k \geqslant \frac{1}{2}(n+3), m=\frac{1}{2}(n-1)$, and $A=C_{m}$.

Case 2. Now suppose for some $m \geqslant\left\lceil\frac{1}{2} n\right\rceil+1$,

$$
A_{m} \neq \varnothing \quad \text { and } \quad A_{m+1}=A_{m+2}=\cdots=A_{n}=\varnothing .
$$

So $m-1 \geqslant n-m+1$, and by Fact 3 with $S=A_{m},|S| \leqslant\left|S_{*}\right|$. Replacing $S$ by $S_{*}$ produces an antichain $A^{\prime}$ at least as large as $A$. Reasoning as in Case 1, this forces $|S|=\left|S_{*}\right|, m=\frac{1}{2}(n+2)$, and $n$ even. Hence, the inequality in Fact 3 is an equality. This implies first that each set $Z \in S$ covers only $m-1$ sets in $S_{*}$, which implies that $|Z \cap Y|=1$ for all $Z \in S$. Secondly, every set in $C_{m}$ covering a set in $S_{*}$ must belong to $S$. By the connectivity of $G_{m-1}$, this forces $S=A=C_{m}$. $|Z \cap Y|=1$ for all $Z \in C_{m}$ implies that $|Y|=1$. Thus $n$ is even, $k=1$, and $A=C_{(1 / 2)(n+2)}$.

Case 3. $A \subseteq C_{\left\lceil\frac{1}{2} n\right\rceil .}$. Clearly $|A|$ is maximum means that $A=C_{\left\lceil\frac{1}{2} n\right\rceil}$.

To complete the proof, observe that for each $n$ and $k$, the remaining possibilities for $A$ have the size required by the theorem.

8. A LYM-style inequality. Lubell [16] discovered the LYM inequality for $B_{n}$ by counting the number of maximal chains in $B_{n}$ passing through an antichain $A$ in $B_{n}$. For such an $A$ the inequality is

$$
\sum_{Z \in A} \frac{1}{\left(\begin{array}{c}
n \\
|Z|
\end{array}\right)} \leqslant 1
$$

In this section Lubell's argument is extended to a wider class of collections of subsets which includes $C(n, k)$. For $0 \leqslant l \leqslant k \leqslant n$ let $C(n, k, l)$ denote the collection of subsets of $X=\{1, \ldots, n\}$ which intersect $Y=\{1, \ldots, k\}$ at least $l$ times. This poset was studied in Example 3.2. Here an LYM-style inequality is derived which is different from the actual LYM inequality for $C(n, k, l)$. 
THEOREM 8.1. Let $A$ be an antichain in $C(n, k, l)$. Then

$$
\sum_{Z \in A} \frac{1}{\left(\begin{array}{c}
n-l \\
|Z|-l
\end{array}\right)} \cdot \frac{\left(\begin{array}{c}
|Z \cap Y| \\
l
\end{array}\right)}{\left(\begin{array}{c}
|Y| \\
l
\end{array}\right)} \leqslant 1 .
$$

Proof. Each maximal chain in $C(n, k, l)$ looks like this: An $l$-subset of $Y$ is at the bottom. The remaining $n-l$ elements of $X$ are joined one at a time, in any order, to produce the other subsets in the chain. Thus there are $\left({ }_{l}^{k}\right)(n-l)$ ! maximal chains.

For $Z \in C(n, k, l)$, a maximal chain through $Z$ is constructed this way: An $l$-subset of $Z \cap Y$ is at the bottom and the remaining elements $Z$ are then joined in any order, to produce $Z$. Then the elements of $X-Z$ are added in any order. So the number of maximal chains through $Z$ is

$$
\left(\begin{array}{c}
|Z \cap Y| \\
l
\end{array}\right)(|Z|-l) !(n-|Z|) ! .
$$

No maximal chain passes through more than one set in $A$. So the sum of the above expressions over all $Z \in A$ is at most the number of maximal chains. This inequality reduces easily to (2).

EXAMPLE 8.1. With $l=0$ we get the usual LYM inequality for $B_{n}$, (1).

EXAMPLE 8.2. With $l=1$ we get this inequality for $C(n, k)$,

$$
\sum_{Z \in A} \frac{1}{\left(\begin{array}{c}
n-1 \\
|Z|-1
\end{array}\right)} \cdot \frac{|Z \cap Y|}{k} \leqslant 1 .
$$

In contrast, the LYM inequality for $C(n, k)$ is

$$
\sum_{Z \in A} \frac{1}{\left(\begin{array}{c}
n \\
|Z|
\end{array}\right)-\left(\begin{array}{c}
n-k \\
|Z|
\end{array}\right)} \leqslant 1 .
$$

Neither inequality appears to imply the other.

\section{REFERENCES}

1. G. F. Clements, More on the generalized Macaulay theorem. II, Discrete Math. 18 (1977), 253-264.

2. E. R. Gansner, On the lattice of order ideals of an up-down poset (preprint).

3. C. Greene and D. J. Kleitman, On the structure of Sperner $k$-families, J. Combin. Theory Ser. A 20 (1976), 41-68.

4. __ Proof techniques in the theory of finite sets, Studies in Combinatorics (G.-C. Rota, editor), Math. Assoc. Amer., 1978, pp. 22-79.

5. __ Strong versions of Sperner's theorem, J. Combin. Theory Ser. A 20 (1976), 80-88.

6. J. R. Griggs, Symmetric chain orders, Sperner theorems, and loop matchings, Ph.D. dissertation, Massachusetts Institute of Technology, 1977.

7. __ On chains and Sperner $k$-families in ranked posets, J. Combin. Theory Ser. A 28 (1980), $156-168$.

8. J. R. Griggs, M. Saks and D. Sturtevant, On chains and Sperner $k$-families in ranked posets. II (to appear).

9. L. H. Harper, The morphology of partially ordered sets, J. Combin. Theory 17 (1974), 44-58.

10. W. N. Hsieh and D. J. Kleitman, Normalized matching in direct products of partial orders, Stud. Appl. Math. 52 (1973), 285-289. 
11. G. O. H. Katona, A theorem of finite sets, Proc. Tihany Conf., 1966, Budapest, 1968.

12. D. J. Kleitman, Extremal hypergraph properties, Surveys in Combinatorics, Proc. 7th British Comb. Conf. (B. Bollobás, editor), London Math. Soc. Lecture Note Series no. 38, 1979.

13. _ On an extremal property of antichains in partial orders: the LYM property and some of its implications and applications, Combinatorics (M. Hall and J. H. van Lint, editors), Math. Centre Tracts, Amsterdam, vol. 55, 1974, pp. 77-90.

14. J. Kruskal, The number of simplices in a complex, Math. Optimization Techniques, Univ. of California Press, Berkeley and Los Angeles, 1963, pp. 251-278.

15. K.-W. Lih, Sperner families over a subset (preprint).

16. D. Lubell, A short proof of Sperner's theorem, J. Combin. Theory 1 (1966), 299.

17. R. A. Proctor, M. Saks and D. Sturtevant, Product partial orders with the Sperner property (to appear).

18. E. Sperner, Ein Satz über Untermengen einer endlichen Menge, Math. Z. 27 (1928), 544-548.

19. A. Wareham, Ph.D. dissertation, University of California, Riverside, 1980.

Department of Mathematics, University of Hawail at Manoa, Honolulu, Hawail 96822

Current address: Department of Mathematics and Statistics, University of South Carolina, Columbia, South Carolina 29208 